\title{
Functional neuroimaging with default mode network regions distinguishes PTSD from TBI in a military veteran population
}

\author{
Cyrus A. Raji ${ }^{1} \cdot$ Kristen Willeumier $^{2} \cdot$ Derek Taylor $^{2} \cdot$ Robert Tarzwell $^{3}$. \\ Andrew Newberg ${ }^{4}$ - Theodore A. Henderson ${ }^{5}$ - Daniel G. Amen ${ }^{2}$
}

Published online: 23 April 2015

(C) The Author(s) 2015. This article is published with open access at Springerlink.com

\begin{abstract}
PTSD and TBI are two common conditions in veteran populations that can be difficult to distinguish clinically. The default mode network (DMN) is abnormal in a multitude of neurological and psychiatric disorders. We hypothesize that brain perfusion SPECT can be applied to diagnostically separate PTSD from TBI reliably in a veteran cohort using DMN regions. A group of 196 veterans (36 with PTSD, 115 with TBI, 45 with PTSD/TBI) were selected from a large multi-site population cohort of individuals with psychiatric disease. Inclusion criteria were peacetime or wartime veterans regardless of branch of service and included those for whom the traumatic brain injury was not service related. SPECT imaging was performed on this group both at rest and during a concentration task. These measures, as well as the baselineconcentration difference, were then inputted from DMN regions into separate binary logistic regression models controlling for age, gender, race, clinic site, co-morbid psychiatric diseases, TBI severity, whether or not the TBI was service related, and branch of armed service. Predicted probabilities were then inputted into a receiver operating characteristic
\end{abstract}

Cyrus A. Raji

cyrusraji@gmail.com

1 Department of Radiology, UCLA Medical Center, 757 Westwood Blvd, Los Angeles, CA 90095, USA

2 Department of Research, Amen Clinics, Inc, Costa Mesa, CA, USA

3 Faculty of Medicine, Department of Psychiatry, University of British Columbia School of Medicine and Clinical Director of Research for Mental Health, Lions Gate Hospital, Vancouver, BC, Canada

4 Department of Radiology, Thomas Jefferson University, Philadelphia, PA, USA

5 The Synaptic Space and The International Society of Applied Neuroimaging, Denver, CO, USA analysis to compute sensitivity, specificity, and accuracy. Compared to PSTD, persons with TBI were older, male, and had higher rates of bipolar and major depressive disorder $(p<0.05)$. Baseline quantitative regions with SPECT separated PTSD from TBI in the veterans with $92 \%$ sensitivity, $85 \%$ specificity, and $94 \%$ accuracy. With concentration scans, there was $85 \%$ sensitivity, $83 \%$ specificity and $89 \%$ accuracy. Baseline-concentration (the difference metric between the two scans) scans were $85 \%$ sensitivity, $80 \%$ specificity, and $87 \%$ accuracy. In separating TBI from PTSD/TBI visual readings of baseline scans had $85 \%$ sensitivity, $81 \%$ specificity, and $83 \%$ accuracy. Concentration scans had $80 \%$ sensitivity, $65 \%$ specificity, and $79 \%$ accuracy. Baseline-concentration scans had $82 \%$ sensitivity, $69 \%$ specificity, and $81 \%$ accuracy. For separating PTSD from PTSD/TBI baseline scans had $87 \%$ sensitivity, $83 \%$ specificity, and $92 \%$ accuracy. Concentration scans had $91 \%$ sensitivity, $76 \%$ specificity, and $88 \%$ accuracy. Baseline-concentration scans had $84 \%$ sensitivity, $64 \%$ specificity, and $85 \%$ accuracy. This study demonstrates the ability to separate PTSD and TBI from each other in a veteran population using functional neuroimaging.

Keywords Functional Neuroimaging · PTSD $\cdot$ SPECT · TBI

\section{Introduction}

Traumatic brain injury (TBI), whether by direct impact or due to blast wave effects, is an all-too-common and devastating feature of armed conflict. With the proliferation of improvised explosive devices (Okie 2005), TBI became the "signature wound" of the conflicts in Afghanistan and Iraq (Snell and Halter 2010). From these conflicts, returning soldiers also report experiencing severe psychological trauma with $50 \%$ witnessing the death or injury of a friend, $10 \%$ had been 
injured themselves, and over $19 \%$ had symptoms consistent with posttraumatic stress disorder (PTSD) (Tanielian et al. 2008). Another study suggests that $90 \%$ of soldiers witnessed a traumatic episode while in service (Hoge et al. 2008). It is therefore not surprising that 400,000 military personnel and veterans have been diagnosed with PTSD or TBI since 2001, and many have been diagnosed with both (Congressional Budget Office 2012).

TBI and PTSD are both common in military populations, making it a challenge to distinguish the two conditions from one another (Hoge et al. 2008). Clinically, these populations may overlap by 33 to $42 \%$ (Lew 2005). A recent study in Veterans Administration facilities revealed $73 \%$ of patients reporting TBI were comorbid for PTSD (Taylor et al. 2012), while $13.5 \%$ of military personnel from recent conflicts reported PTSD (Dursa et al. 2014). For patients who have both TBI and PTSD, which generate many of the same symptoms, the VA acknowledges that the patient is often diagnosed with one or the other. Note that several of the question items within the Clinician-Administered PTSD scale (Blake et al. 1998) can be symptoms of PTSD, such as poor concentration, memory difficulties, anhedonia, social isolation, sleep difficulties, and irritability. The VA recently concluded there was a lack of diagnostic accuracy for the dually affected veteran (Congressional Budget Office 2012). Assessment of an event can be further confounded because memories of traumatic experiences, particularly long after the actual event occurred, can be inconsistent. Lastly, there is often a mismatch between performance on neuropsychological testing and the patient's experience of the deficits in those with mild TBI, which is the most common form of TBI (Brenner et al. 2010). Some evidence suggests that additional recruitment of cortical areas during a task contributes to performance on neuropsychological testing in those with TBI (Van Boven et al. 2009).

The currently available treatments of PTSD and TBI are different. Moreover, the treatments for PTSD may be harmful or, at best, not helpful in the case of TBI. While the wellestablished PTSD treatments of exposure therapy (Rauch et al. 2012; Schnyder 2014), cognitive processing therapy (Walter et al. 2014) and trauma-focused cognitive behavioral therapy (Schnyder 2014) are helpful in PTSD, there is no evidence to suggest that they would be helpful for those with mild TBI who are misdiagnosed as having PTSD. The pharmacological treatments for PTSD include the serotonin reuptake inhibitors, serotonin-norepinephrine reuptake inhibitors, benzodiazepines, mood stabilizers, and atypical antipsychotics (Jain et al. 2012; Watts et al. 2013). While antidepressants can be useful in managing some of the symptoms of TBI, the prescribing of benzodiazepines to those with TBI can impede function or be dangerous (Silver et al. 2011;
Kennedy et al. 2007; Morgan et al. 2012). Similarly, antipsychotics are often prescribed for PTSD (Morgan et al. 2012) and clinical studies support the use of atypical antipsychotics (Wang et al. 2013; Hermes et al. 2014; Krystal et al. 2011); however, antipsychotics have been shown to impede recovery or be dangerous in clinical studies and animal models of TBI (Kline et al. 2008; Phelps et al. 2014). Curiously, having a diagnosis of TBI increases the likelihood that a veteran will be prescribed a benzodiazepine or an antipsychotic. While $41 \%$ of veterans with PTSD were prescribed benzodiazepines, those with both PTSD and TBI had a $67 \%$ chance of being prescribed a benzodiazepine. Antipsychotics were prescribed to $25 \%$ of veterans with PTSD, but $40 \%$ of those with both PTSD and TBI (Morgan et al. 2012). Other treatments for PTSD, such as transcranial magnetic stimulation (Berlim and Van Den Eynde 2014), can be dangerous in TBI due to induction of seizures (Castel-Lacanal et al. 2014). Emerging treatments for TBI are more targeted and require an understanding of what portion of the individual's brain is involved, while there are evidence-based, well-established treatments for PTSD (Ursano et al. 2004). Therefore, where clinical uncertainty exists, an accurate biomarker for each of these conditions may be helpful in providing diagnostic clarity that would then allow initiation of an appropriate treatment algorithm.

The default mode network (DMN), consisting of the inferior orbital frontal cortex, anterior cingulate gyrus, posterior cingulate gyrus, hippocampus, precuneus, superior parietal lobe, and angular gyrus (Buckner et al. 2008), shows hyperperfusion in patients diagnosed with PTSD and hypoperfusion in patients diagnosed with TBI (Erickson et al. 2014; Dubroff and Newberg 2008; Newberg and Alavi 2003; Newberg et al. 2014; Liu et al. 2013). By further elucidating the correlation between increased perfusion in DMN structures in PTSD and decreased perfusion in TBI, clinicians may be able to more reliably diagnose PTSD from TBI.

Brain single photon emission computed tomography (SPECT) is a widely available functional neuroimaging study indicated for the evaluation of TBI in the absence of anatomical findings (Davis et al. 2000). A recent review of three decades of research (Raji et al. 2014) supports the diagnostic utility of SPECT in TBI. The purpose of this study was to assess the DMN using quantitative SPECT to assess whether PTSD can be separated from TBI in a veteran population. In particular, brain regions associated with DMN were analyzed using SPECT to determine whether there were significant differences between individuals diagnosed with PTSD and those diagnosed with TBI. Because previous literature suggests that PTSD patients have increased perfusion of DMN (Philip et al. 2014; Shin et al. 1999), while TBI patients have decreased perfusion of DMN, DMN perfusion SPECT data could act as a biomarker in the differentiation of the two diseases. Therefore, brain perfusion SPECT could provide, in cases of clinical uncertainty, an accurate diagnosis and guide patients 
with PTSD or TBI toward appropriate treatment by examining the status of DMN perfusion in the baseline state.

\section{Methods}

\section{Study subjects}

All subjects were obtained for retrospective analysis from a large multisite psychiatric database involving 20,746 patients who came for evaluation of complex, treatment resistant issues to one of nine outpatient clinics (Newport Beach, Costa Mesa, Fairfield, and Brisbane, CA, Tacoma and Bellevue, WA, Reston, VA, Atlanta, GA and New York, NY) between 1995 and 2014. Diagnoses were made by board certified or eligible psychiatrists, using all of the data available to them, including detailed clinical history, mental status examination and DSM-IV or V criteria, consistent with the current standard of care. Anonymous data was mined from a research database containing SPECT data and corresponding clinical information (IRB \#004). This retrospective review was approved by an accredited institutional review board IntegReview (http:// www.integreview.com/). Specifically, peacetime or combat veterans with either a DSM-IV diagnosis of PSTD, TBI, or those with PTSD and TBI were selected for this analysis. These inclusion and exclusion criteria yielded a total of 196 subjects (115 TBI, 36 PTSD, and 45 with PTSD and TBI). Subject characteristics are displayed in Table 1. All individuals in this study were right handed. No subjects with epilepsy, a condition that can produce dramatic focal SPECT abnormalities, were included in the analysis. Subjects with history of stroke were also excluded from the study. No sedating or stimulating drugs such as caffeine were consumed on the day of the SPECT examination per Society for Nuclear Medicine scanning guidelines for SPECT scanning (Juni et al. 1998, 2009; Kapucu et al. 2009).

Among the TBI group, $53 \%$ had mild TBI, $4.3 \%$ had moderate TBI, and $3.5 \%$ had severe TBI. There were $38.3 \%$ who had an unknown severity of TBI. Psychiatric comorbidity was similar to other populations of veterans with complex issues (Bowe and Rosenheck 2015). With respect to the veteran demographics by service, $41 \%$ were Army, $17 \%$ were Marines, $19 \%$ were Air Force, and $23 \%$ were Navy. Of the subjects with TBI, $19 \%$ were associated with an event that occurred during service.

\section{SPECT imaging}

SPECT was obtained in conjunction with clinical assessments before any intervening treatment. SPECT was performed as previously described (Amen et al. 2008a, b). For each procedure, an age- and weight-appropriate dose of technetium-99m exametazime was administered intravenously at rest and while performing a concentration task. For the baseline scans, patients were injected while they sat in a dimly lit room with eyes open. Patients were scanned approximately $30 \mathrm{~min}$ after injection. For the concentration scans, patients were injected three minutes after starting the Conners Continuous Performance Test (C-CPT). Approximately $30 \mathrm{~min}$ after the injection, subjects were scanned. Photon emission was captured using a high resolution Picker (Phillips) Prism XP 3000 triple-headed gamma camera with fan beam collimators with data collected in 128x128 matrices, yielding 120 images per scan with each image separated by three degrees spanning 360 degrees. A low pass filter was applied with a high cutoff. Chang attenuation correction was performed (Chang 1977, 1984). Transaxial slices oriented horizontal to the AC-PC line were created along with coronal and sagittal images $(6.6 \mathrm{~mm}$

Table 1 Subject characteristics

\begin{tabular}{|c|c|c|c|c|}
\hline Variable & $\operatorname{PTSD}(n=36)$ & TBI $(n=115)$ & TBI/PTSD $(n=45)$ & $\begin{array}{l}\text { TBI compared to PTSD; PTSD/TBI } \\
\text { compared to PTSD or TBI ( } \chi \text { or } \mathrm{t}, p \text {-value })\end{array}$ \\
\hline Age & $45.8 \pm 15.4$ & $38.6 \pm 12$ & $42 \pm 14.4$ & $-2.5,0.01 ;-0.8,0.4$ \\
\hline Gender \%Male (n) & $69(25)$ & $94(108)$ & $78(35)$ & $15,<0.001 ; 3.1,0.09$ \\
\hline Race \% Caucasian (n) & $75(27)$ & $77(89)$ & $84(38)$ & $0.35,0.49 ; 4.8,0.7$ \\
\hline Dementia\% (n) & $3(1)$ & $5.2(6)$ & $4(2)$ & $0.09,0.67 ; 0.00,1$ \\
\hline Depression\% (n) & $33(12)$ & $50(58)$ & $36(16)$ & $3.7,0.04 ; 0.02,0.87$ \\
\hline Schizophrenia\% (n) & $3(1)$ & $2.8(3)$ & $0(0)$ & $0.17,0.56 ; 1.8,0.34$ \\
\hline ADHD\% (n) & $70(25)$ & $56(64)$ & $71(32)$ & $2.4,0.09 ; 0.34,0.55$ \\
\hline Bipolar\% (n) & $3(1)$ & $14(16)$ & $11(5)$ & $5.3,0.04 ; 1.4,0.25$ \\
\hline Substance Abuse\% (n) & $25(9)$ & $17(20)$ & $27(12)$ & $0.74,0.27 ; 0.48,0.54$ \\
\hline Alcohol Use\% (n) & $14(5)$ & $6(7)$ & $13(6)$ & $1.2,0.36 ; 0.27,0.59$ \\
\hline Location \%Newport (n) & $36(13)$ & $39(45)$ & $36(16)$ & $0.106,0.84 ; 5.2,0.22$ \\
\hline
\end{tabular}


apart, unsmoothed). Three dimensional reformats were generated for review based on Odyssey image visualization software.

\section{SPECT region of interest analysis}

Bilateral ROI counts were derived from the anatomical regions in the Automated Anatomical Labeling (AAL) atlas (TzourioMazoyer et al. 2002). These quantitative ROI metrics were in no way used to aid in the clinical diagnosis of PTSD or TBI. To account for outliers, T-score derived ROI count measurements were derived using trimmed means (Thomas and Tilanus1964) that are calculated using all scores within the $98 \%$ confidence interval $(-2.58<\mathrm{Z}<2.58)$. The ROI mean for each subject and the trimmed mean for the sample are used to calculate $\mathrm{T}$ with the following formula: $\mathrm{T}=10^{*}($ (subject ROI mean - trimmed regional_avg)/trimmed regional_stdev) +50 .

\section{Statistical analyses}

All analyses were performed using Statistical Package for Social Science (SPSS, version 22, IBM, Armonk, NY). Multiple imputations identified less than $10 \%$ missing data in the analysis. Default mode network regions as defined (Raichle et al. 2001; Buckner et al. 2008) were inputted into a binary logistic regression analysis controlling for comorbidities and demographics as defined in Table 1 and also controlling for TBI severity and armed service branch. The default mode network regions selected were: bilateral angular gyrus, anterior and posterior cingulate gyri, the precuneus, inferior orbital frontal cortex, superior parietal lobe, hippocampus, and parahippocampal gyrus. Both baseline and concentration regions were selected for separate binary logistic regression analyses. Additionally, the difference between baseline and concentration regions was also inputted into a separate analysis. For each analysis, predicted probabilities were extracted from this analysis and then inputted into a receiver operating characteristic curve to calculate sensitivity (the true positive results as a fraction of the true positives plus false negatives), specificity (the true negative results as a fraction of false positives plus true negatives), and accuracy (the area under the curve defined as true positives plus true negatives divided by total sample).

\section{Results}

Quantitative SPECT imaging of DMN regions, at baseline or on concentration task, distinguished veterans with PTSD from those with TBI with an accuracy range of 87-94 \%. These measures can delineate TBI from those with PTSD and TBI with an accuracy range of 79-83\%.
For distinguishing veterans with PTSD from those with both, the accuracy range is $85-92 \%$.

Table 2 details the diagnostic utility of baseline, concentration, and baseline-concentration default mode network regions in distinguishing TBI from PTSD:

Figure 1 shows a summary of the diagnostic utility of brain SPECT in distinguishing PTSD from TBI. Figure 2 shows two groups of ROC curves. Figure 2a shows ROC curves delineating veterans with PTSD from those with both PTSD and TBI. Figure $2 \mathrm{~b}$ displays ROC curves distinguishing veterans with TBI from those with PTSD and TBI. Figure 3 shows two rows of volume rendered SPECT images, each from separate subjects. The first volume rendered row shows inferior underside surface rendered images. The second row shows intensity projection images in which white colors represent the top $8 \%$ and red colors represent the top $15 \%$ of cerebral flow in that subject's brain compared to their whole brain perfusion. A healthy control shows normal higher perfusion to the cerebellum. The PTSD subject shows increased perfusion in the brain - particularly in the frontal lobes. The TBI subject shows decreased perfusion throughout by comparison. The subject with both PTSD and TBI shows perfusion that is intermediate in that it is lower than the person with PTSD but higher than the subject with TBI. These representative images highlight how, in a given set of subjects, there is increased perfusion in PTSD and hypoperfusion in TBI with an intermediate imaging pattern in persons with both.

\section{Discussion}

This study identified regions that distinguished TBI patients from those with PTSD. Because quantitative ROIs were not used in any way to initially establish the clinical diagnoses of TBI and PTSD, they serve as a particularly rigorous

Table 2 Diagnostic utility of quantitative brain SPECT default mode network

\begin{tabular}{llll}
\hline Variable & Sensitivity & Specificity & $\begin{array}{l}\text { Accuracy } \\
(95 \% \mathrm{CI})\end{array}$ \\
\hline $\begin{array}{l}\text { PTSD from TBI } \\
\quad \text { Baseline }\end{array}$ & 92 & 85 & $94(88-99)$ \\
Concentration & 85 & 83 & $89(83-95)$ \\
$\quad$ Baseline-Concentration & 85 & 80 & $87(79-95)$ \\
PTSD/TBI from TBI & & & \\
Baseline & 85 & 81 & $83(76-90)$ \\
Concentration & 80 & 65 & $79(81-88)$ \\
Baseline-Concentration & 82 & 69 & $81(74-88)$ \\
PTSD/TBI from PTSD & & & $92(86-99)$ \\
BL & 87 & 83 & $88(81-96)$ \\
Conc & 91 & 76 & $85(75-94)$ \\
BL-Conc & 84 & 64 & \\
\hline
\end{tabular}


a

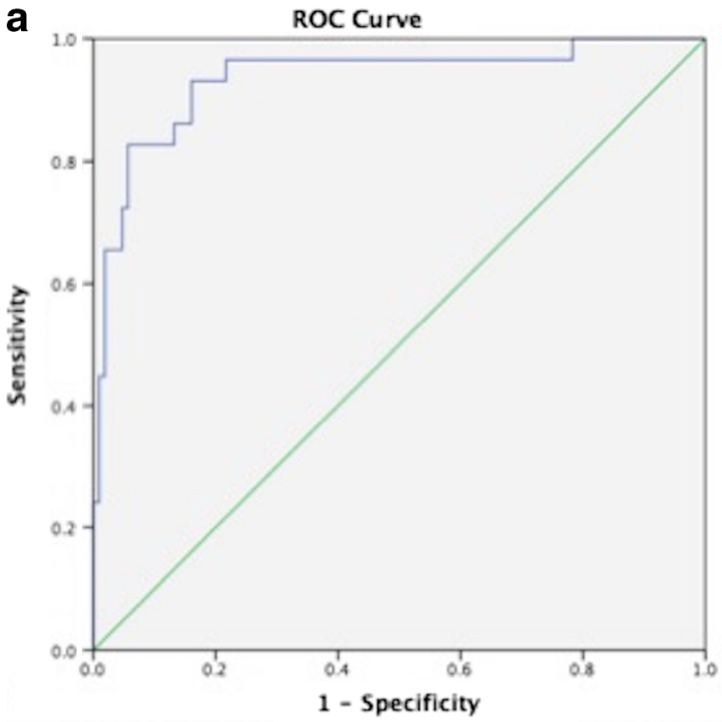

b

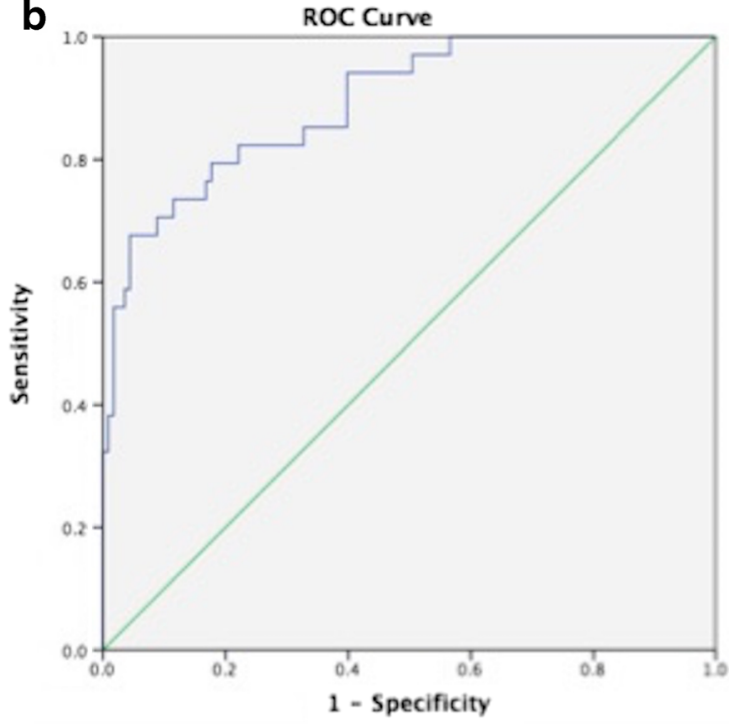

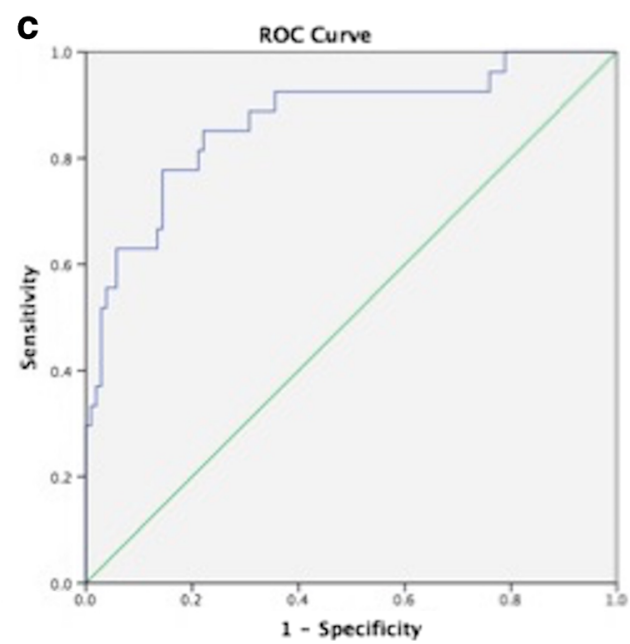

Fig. 1 Receiver operating characteristic curves for quantitative baseline (a), concentration (b) and (Baseline-Concentration) (c) default mode network region in separating PTSD from TBI

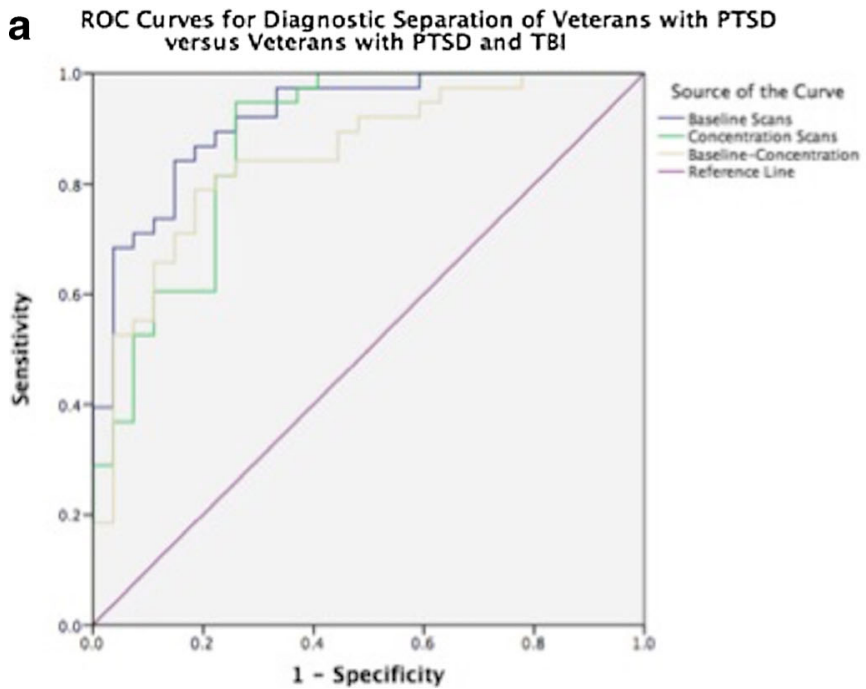

b ROC Curves for Diagnostic Separation of Veterans with TBI
versus Veterans with PTSO and TBI

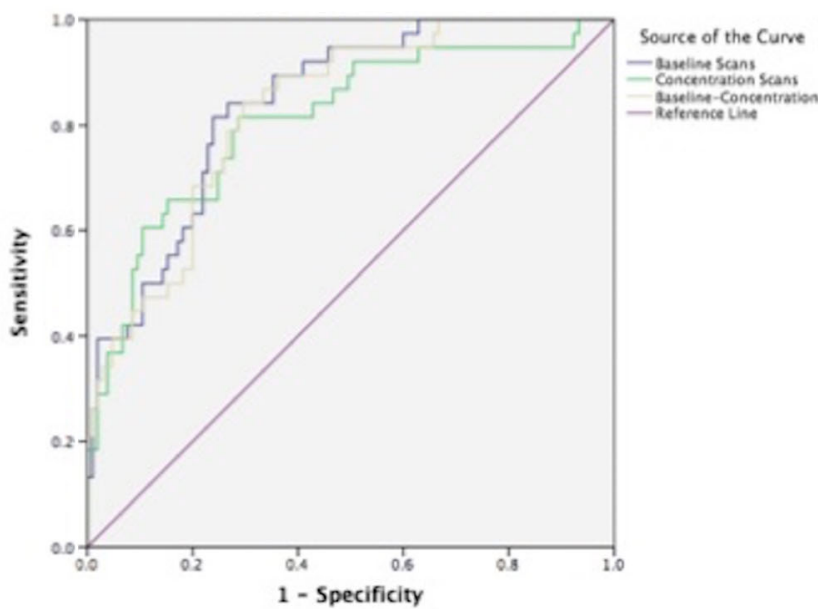

Fig. 2 a Shows ROC curves delineating veterans with PTSD from those with both PTSD and TBI. b Displays ROC curves distinguishing veterans with TBI from those with PTSD and TBI 
Fig. 3 Healthy vs Classic PTSD vs TBI vs Both

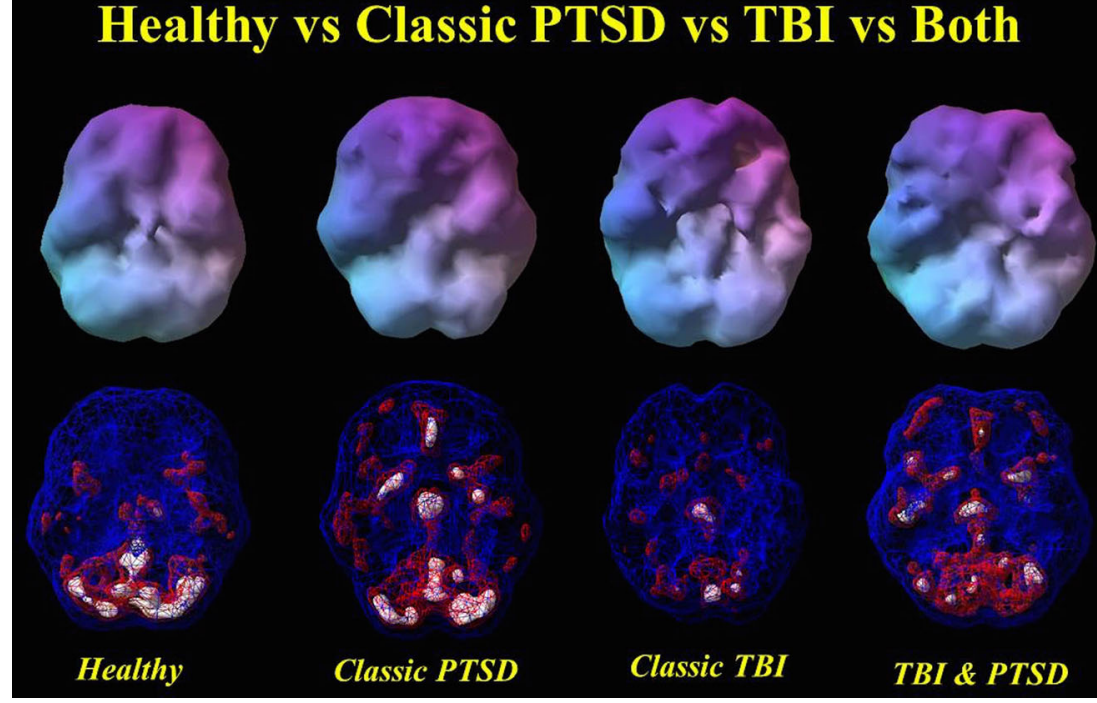

independent predictor of diagnostic category. Use of the DMN for discriminating TBI from PTSD in baseline versus concentration states further expands upon the evolving concept of applying these regions in better understanding brain health and disease (Raichle et al. 2001; Morcom and Fletcher 2007). SPECT imaging can help distinguish PTSD and TBI with good sensitivity and specificity on both baseline and concentration scans. These data demonstrate that SPECT may be a reasonably sensitive test, with accuracy in a clinically relevant range for distinguishing TBI from PTSD in veterans. SPECT data in the study show that elements of the DMN are affected in both TBI and PTSD, but in an opposite manner. Specifically, the DMN is hyperperfused in individuals diagnosed with PTSD and hypoperfused in patients diagnosed with TBI. The data suggests that a specific neural network, the DMN, is implicated in both PTSD and TBI contributing to the similarity in symptoms. However, the pathophysiologies of the diseases are different in the way they effect perfusion of DMN. SPECT may therefore allow clinicians to differentially diagnose PTSD and TBI. While the data suggest that baseline SPECT alone may be sufficient for delineation of PTSD versus TBI, concentration SPECT may be useful in further refining the neurophysiological deficits in persons for which their TBI or PTSD may manifest with hypo or hyperattentive symptoms. Given the wide availability of brain SPECT imaging and the need for accurate diagnosis in distinguishing PTSD from TBI, if this test retains accuracy in prospective trials, it could be translated into the clinical care of veteran population.

Peterson's recent survey takes a network-based approach to findings in $11 \mathrm{fMRI}$ studies, which met her quality threshold over the survey period, 2009 to mid-2013 (Peterson et al. 2014). They report a positive correlation between DMN activity in PTSD severity in five studies, negative in two, suggesting that DMN overactivity may be responsible for symptoms associated with PTSD. DMN activity and PTSD symptom severity have been shown to correlate positively (Lanius et al. 2010) while DMN activity and TBI have been shown to correlate negatively (Bonnelle et al. 2011). DMN is activated when individuals are performing internally focused tasks, which include the recall of autobiographical memories, the consideration of the thoughts and feelings of others, and attention attenuation (Sandrone and Bacigaluppi 2012). This data further corroborates the existing evidence on DMN alterations that differentially influence persons with PTSD versus TBI. Taken together, the results of this study suggest that DMN SPECT perfusion data may act as a reasonably accurate biomarker to differentially diagnose PTSD and TBI as has been done in other conditions such as dementia (Henderson2012).

A subtle, but critical, aspect of neuroimaging is the ability to show the patient a picture of what is occuring in their brain. Only one-quarter to one-third of soldiers and veterans with PTSD who screened positive for PTSD receive treatment (Hoge et al. 2014; Stecker et al. 2013, 2014). Guilt, shame, fears of medications, lack of confidence in the therapies offered, and denial of a medical problem are among the reasons treatment is avoided even though efficacious medications and psychotherapy are available for PTSD and correct diagnosis will facilitate use of evidence-based practices (Hoge et al. 2014; Stecker et al. 2013, 2014; Murphy et al. 2014; Bryan et al. 2013). The barriers to treatment for patients with TBI are unknown, but can be suspected to be similar. Herein, we show that the functional brain scan can provide a depiction of the biological processes underlying a patient's symptoms, which can reduce stigma, foster efforts to seek a medical solution, and remove self-blame/shame.

Advantages of this study include the application of a quantitative functional neuroimaging methodology to a wellcharacterized veteran cohort. An additional advantage was a 
multivariable approach in the logistic modeling that controlled for multiple other factors including psychiatric co-morbidities. The main caveat of this study is its retrospective nature. Future work will benefit from a prospective approach to better understand how imaging data may improve patient outcomes. Functional neuroimaging with SPECT can be used to differentiate PTSD and TBI by examining the activity of DMN in the baseline state. By examining the difference in perfusion of DMN using SPECT, veterans can be better assessed for the purpose of diagnostically separating TBI from PTSD. Given the wide availability of SPECT and the existence of internationally accepted standards in nuclear medicine for performing baseline state perfusion scans (Kapucu et al. 2009; Juni et al. 2009), the potential for rapid clinical translation makes this test especially significant in the clinical care of persons with PTSD or TBI.

Conflict of Interest Cyrus A. Raji, Kristen Willeumier, Derek Taylor, Robert Tarzwell, Andrew Newberg, Theodore A. Henderson, and Daniel G. Amen declare that they have no conflicts of interest.

Open Access This article is distributed under the terms of the Creative Commons Attribution 4.0 International License (http:// creativecommons.org/licenses/by/4.0/), which permits unrestricted use, distribution, and reproduction in any medium, provided you give appropriate credit to the original author(s) and the source, provide a link to the Creative Commons license, and indicate if changes were made.

\section{References}

Amen, D. G., Hanks, C., \& Prunella, J. (2008a). Predicting positive and negative treatment responses to stimulants with brain SPECT imaging. Journal of Psychoactive Drugs, 40, 131-138.

Amen, D. G., Hanks, C., \& Prunella, J. (2008b). Preliminary evidence differentiating ADHD using brain SPECT imaging in older patients. Journal of Psychoactive Drugs, 40, 139-146.

Berlim, M. T., \& Van Den Eynde, F. (2014). Repetitive transcranial magnetic stimulation over the dorsolateral prefrontal cortex for treating posttraumatic stress disorder: an exploratory meta-analysis of randomized, double-blind and sham-controlled trials. Canadian Journal of Psychiatry. Revue Canadienne de Psychiatrie, 59, 487496.

Blake D. D., Weathers F. W., Nagy L. M., Kaloupek D. G., Charney D. S., Keane T. M. (1998). Available at: http://www.clintools.com/victims/ resources/assessment/ptsd/protected/CAPSIV.pdf. Accessed February 2nd.

Bonnelle, V., Leech, R., Kinnunen, K. M., et al. (2011). Default mode network connectivity predicts sustained attention deficits after traumatic brain injury. The Journal of Neuroscience: The Official Journal of the Society for Neuroscience, 31, 13442-13451.

Bowe, A., \& Rosenheck, R. (2015). PTSD and substance use disorder among veterans: characteristics, service utilization and pharmacotherapy. Journal of Dual Diagnosis, 11, 22-32.

Brenner, L. A., Terrio, H., Homaifar, B. Y., et al. (2010). Neuropsychological test performance in soldiers with blast-related mild TBI. Neuropsychology, 24, 160-167.

Bryan, C. J., Morrow, C. E., Etienne, N., \& Ray-Sannerud, B. (2013). Guilt, shame, and suicidal ideation in a military outpatient clinical sample. Depression and Anxiety, 30, 55-60.
Buckner, R. L., Andrews-Hanna, J. R., \& Schacter, D. L. (2008). The brain's default network: anatomy, function, and relevance to disease. Annals of the New York Academy of Sciences, 1124, 1-38.

Castel-Lacanal, E., Tarri, M., Loubinoux, I., et al. (2014). Transcranial magnetic stimulation in brain injury. Annales Françaises d'Anesthèsie et de Rèanimation, 33, 83-87.

Chang, L. T. (1977). A method for attenuation correction in radionuclide computed tomography. IEEE Transactions on Nuclear Science, 25, 638-643.

Chang, W., Henkin, R. E., \& Buddemeyer, E. (1984). The sources of overestimation in the quantification by SPECT of uptakes in a myocardial phantom: concise communication. Journal of Nuclear Medicine: Official Publication, Society of Nuclear Medicine, 25, 788-791.

Congressional Budget Office(2012). The Veterans Health Administration's Treatment of PTSD and Traumatic Brain Injury among Recent Combat Veterans. The Congress of the United States, Washington, D.C.

Davis, P. C., Drayer, B. P., Anderson, R. E., et al. (2000). Head trauma. American college of radiology. ACR appropriateness criteria. Radiology, 215(Suppl), 507-524.

Dubroff, J. G., \& Newberg, A. (2008). Neuroimaging of traumatic brain injury. Seminars in Neurology, 28, 548-557.

Dursa, E. K., Reinhard, M. J., Barth, S. K., \& Schneiderman, A. I. (2014). Prevalence of a positive screen for PTSD among OEF/OIF and $\mathrm{OEF} / \mathrm{OIF}-\mathrm{Era}$ veterans in a large population-based cohort. Journal of Traumatic Stress, 27, 542-549.

Erickson, H. J., Hurley, R. A., \& Taber, K. (2014). Psychotherapy for PTSD: neuroimaging of recovery processes. The Journal of Neuropsychiatry and Clinical Neurosciences, 26, 188-195.

Henderson, T. A. (2012). The diagnosis and evaluation of dementia and mild cognitive impairment with emphasis on SPECT perfusion neuroimaging. CNS Spectrums, 17, 176-206.

Hermes, E., Sernyak, M., \& Rosenheck, R. (2014). The use of second generation antipsychotics for post-traumatic stress disorder in a US Veterans health administration medical center. Epidemiology and Psychiatric Sciences, 23, 281-288.

Hoge, C. W., McGurk, D., Thomas, J. L., Cox, A. L., Engel, C. C., \& Castro, C. A. (2008). Mild traumatic brain injury in U.S. Soldiers returning from Iraq. The New England Journal of Medicine, 358, 453-463.

Hoge, C. W., Grossman, S. H., Auchterlonie, J. L., Riviere, L. A., Milliken, C. S., \& Wilk, J. E. (2014). PTSD treatment for soldiers after combat deployment: low utilization of mental health care and reasons for dropout. Psychiatric Services, 65, 997-1004.

Jain, S., Greenbaum, M. A., \& Rosen, C. (2012). Concordance between psychotropic prescribing for veterans with PTSD and clinical practice guidelines. Psychiatric Services, 63, 154-160.

Juni, J. E., Waxman, A. D., Devous, M. D., Sr., et al. (1998). Procedure guideline for brain perfusion SPECT using technetium-99 $\mathrm{m}$ radiopharmaceuticals. Society of nuclear medicine. Journal of Nuclear Medicine: Official Publication, Society of Nuclear Medicine, 39, 923-926.

Juni, J. E., Waxman, A. D., Devous, M. D., Sr., et al. (2009). Procedure guideline for brain perfusion SPECT using $(99 \mathrm{~m}) \mathrm{Tc}$ radiopharmaceuticals 3.0. Journal of Nuclear Medicine Technology, 37, 191195.

Kapucu, O. L., Nobili, F., Varrone, A., et al. (2009). EANM procedure guideline for brain perfusion SPECT using 99mTc-labelled radiopharmaceuticals, version 2. European Journal of Nuclear Medicine and Molecular Imaging, 36, 2093-2102.

Kennedy, J. E., Jaffee, M. S., Leskin, G. A., Stokes, J. W., Leal, F. O., \& Fitzpatrick, P. J. (2007). Posttraumatic stress disorder and posttraumatic stress disorder-like symptoms and mild traumatic brain injury. Journal of Rehabilitation Research and Development, 44, 895-920. 
Kline, A. E., Hoffman, A. N., Cheng, J. P., Zafonte, R. D., \& Massucci, J. L. (2008). Chronic administration of antipsychotics impede behavioral recovery after experimental traumatic brain injury. Neuroscience Letters, 448, 263-267.

Krystal, J. H., Rosenheck, R. A., Cramer, J. A., et al. (2011). Adjunctive risperidone treatment for antidepressant-resistant symptoms of chronic military service-related PTSD: a randomized trial. JAMA, the Journal of the American Medical Association, 306, 493-502.

Lanius, R. A., Bluhm, R. L., Coupland, N. J., et al. (2010). Default mode network connectivity as a predictor of post-traumatic stress disorder symptom severity in acutely traumatized subjects. Acta Psychiatrica Scandinavica, 121, 33-40.

Lew, H. L. (2005). Rehabilitation needs of an increasing population of patients: traumatic brain injury, polytrauma, and blast-related injuries. Journal of Rehabilitation Research and Development, 42, xii-xvi.

Liu, W., Wang, B., Wolfowitz, R., et al. (2013). Perfusion deficits in patients with mild traumatic brain injury characterized by dynamic susceptibility contrast MRI. NMR in Biomedicine, 26, 651-663.

Morcom, A. M., \& Fletcher, P. C. (2007). Does the brain have a baseline? Why we should be resisting a rest. NeuroImage, 37, 1073-1082.

Morgan, M., Lockwood, A., Steinke, D., Schleenbaker, R., \& Botts, S. (2012). Pharmacotherapy regimens among patients with posttraumatic stress disorder and mild traumatic brain injury. Psychiatric Services, 63, 182-185.

Murphy, D., Hunt, E., Luzon, O., \& Greenberg, N. (2014). Exploring positive pathways to care for members of the UK armed forces receiving treatment for PTSD: a qualitative study. European Journal of Psychotraumatology, 5, 1-8.

Newberg, A. B., \& Alavi, A. (2003). Neuroimaging in patients with head injury. Seminars in Nuclear Medicine, 33, 136-147.

Newberg, A. B., Serruya, M., Gepty, A., et al. (2014). Clinical comparison of 99mTc exametazime and 123I Ioflupane SPECT in patients with chronic mild traumatic brain injury. PloS One, 9, e87009.

Okie, S. (2005). Traumatic brain injury in the war zone. The New England Journal of Medicine, 352, 2043-2047.

Peterson, A., Thome, J., Frewen, P., \& Lanius, R. A. (2014). Resting-state neuroimaging studies: a new way of identifying differences and similarities among the anxiety disorders? Canadian Journal of Psychiatry. Revue Canadienne de Psychiatrie, 59, 294-300.

Phelps T. I., Bondi C. O., Ahmed R. H, Olugbade Y. T., Kline A. E. (2014). Divergent long-term consequences of chronic treatment with haloperidol, risperidone, and bromocriptine on traumatic brain injury-induced cognitive deficits. Journal of Neurotrauma.

Philip N. S., Carpenter S. L, Sweet L. H. (2014). Developing neuroimaging phenotypes of the default mode network in PTSD: integrating the resting state, working memory, and structural connectivity. Journal of Visualized Experiments

Raichle, M. E., MacLeod, A. M., Snyder, A. Z., Powers, W. J., Gusnard, D. A., \& Shulman, G. L. (2001). A default mode of brain function. Proceedings of the National Academy of Sciences of the United States of America, 98, 676-682.

Raji, C. A., Tarzwell, R., Pavel, D., et al. (2014). Clinical utility of SPECT neuroimaging in the diagnosis and treatment of traumatic brain injury: a systematic review. PloS One, 9, e91088.

Rauch, S. A., Eftekhari, A., \& Ruzek, J. I. (2012). Review of exposure therapy: a gold standard for PTSD treatment. Journal of Rehabilitation Research and Development, 49, 679-687.
Sandrone, S., \& Bacigaluppi, M. (2012). Learning from default mode network: the predictive value of resting state in traumatic brain injury. The Journal of Neuroscience: The Official Journal of the Society for Neuroscience, 32, 1915-1917.

Schnyder, U. (2014). Treating intrusions, promoting resilience: an overview of therapies for trauma-related psychological disorders. European Journal of Psychotraumatology, 5, 26520.

Shin, L. M., McNally, R. J., Kosslyn, S. M., et al. (1999). Regional cerebral blood flow during script-driven imagery in childhood sexual abuse-related PTSD: a PET investigation. The American Journal of Psychiatry, 156, 575-584.

Silver, J.M., Arciniegas, D. B., Yudofsky, S. C. (2011). Psychopharmacology. In: Silver J. M., McAllister, T. W., Yudofsky, S. C. (Eds.), Textbook of Traumatic Brain Injury. 2nd edn. (609-639).Arlington, VA: American Psychiatric Publishing.

Snell, F. I., \& Halter, M. J. (2010). A signature wound of war: mild traumatic brain injury. Journal of Psychosocial Nursing and Mental Health Services, 48, 22-28.

Stecker, T., Shiner, B., Watts, B. V., Jones, M., \& Conner, K. R. (2013). Treatment-seeking barriers for veterans of the Iraq and Afghanistan conflicts who screen positive for PTSD. Psychiatric Services, 64, 280-283.

Stecker, T., McHugo, G., Xie, H., Whyman, K., \& Jones, M. (2014). RCT of a brief phone-based CBT intervention to improve PTSD treatment utilization by returning service members. Psychiatric Services, 65, 1232-1237.

Tanielian, T. L., Jaycox, L., \& Rand Corporation. (2008). Invisible wounds of war: psychological and cognitive injuries, their consequences, and services to assist recovery. Santa Monica, CA: RAND.

Taylor, B. C., Hagel, E. M., Carlson, K. F., et al. (2012). Prevalence and costs of co-occurring traumatic brain injury with and without psychiatric disturbance and pain among Afghanistan and Iraq war veteran V.A. users. Medical Care, 50, 342-346.

Thomas, J. F. F. M., \& Tilanus, C. B. (1964). A note on the estimation of the location parameters of the Cauchy distribution. Journal of the American Statistical Association, 61, 852-855.

Tzourio-Mazoyer, N., Landeau, B., Papathanassiou, D., et al. (2002). Automated anatomical labeling of activations in SPM using a macroscopic anatomical parcellation of the MNI MRI single-subject brain. NeuroImage, 15, 273-289.

Ursano, R. J., Bell, C., Eth, S., et al. (2004). Practice guideline for the treatment of patients with acute stress disorder and posttraumatic stress disorder. The American Journal of Psychiatry, 161, 3-31.

Van Boven, R. W., Harrington, G. S., Hackney, D. B., et al. (2009). Advances in neuroimaging of traumatic brain injury and posttraumatic stress disorder. Journal of Rehabilitation Research and Development, 46, 717-757.

Walter, K. H., Dickstein, B. D., Barnes, S. M., \& Chard, K. M. (2014). Comparing effectiveness of CPT to CPT-C among U.S. Veterans in an interdisciplinary residential PTSD/TBI treatment program. Journal of Traumatic Stress, 27, 438-445.

Wang, H. R., Woo, Y. S., \& Bahk, W. M. (2013). Atypical antipsychotics in the treatment of posttraumatic stress disorder. Clinical Neuropharmacology, 36, 216-222.

Watts, B. V., Schnurr, P. P., Mayo, L., Young-Xu, Y., Weeks, W. B., \& Friedman, M. J. (2013). Meta-analysis of the efficacy of treatments for posttraumatic stress disorder. The Journal of Clinical Psychiatry, 74, e541-550. 